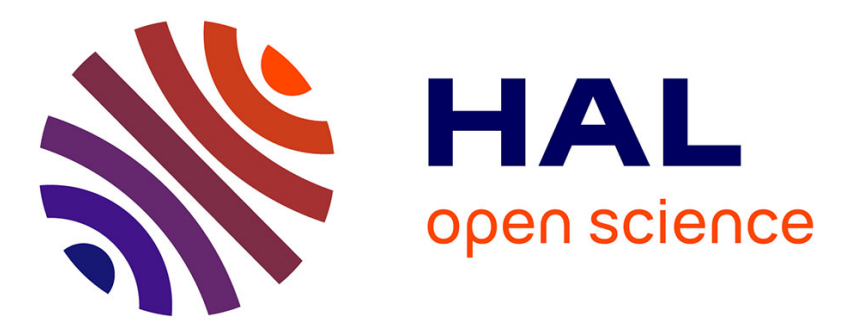

\title{
Scaling-down lithographic dimensions with block-copolymer materials: 10nm-sized features with PS-b-PMMA
}

\author{
X. Chevalier, C. Nicolet, R. Tiron, A. Gharbi, M. Argoud, J. Pradelles, M. \\ Delalande, G. Cunge, G. Fleury, G. Hadziioannou, et al.
}

\section{To cite this version:}

X. Chevalier, C. Nicolet, R. Tiron, A. Gharbi, M. Argoud, et al.. Scaling-down lithographic dimensions with block-copolymer materials: 10nm-sized features with PS-b-PMMA. SPIE Advanced Lithography, 2013, San Diego, United States. 10.1117/12.2011405 . hal-00944986

\section{HAL Id: hal-00944986 https://hal.science/hal-00944986}

Submitted on 19 Mar 2020

HAL is a multi-disciplinary open access archive for the deposit and dissemination of scientific research documents, whether they are published or not. The documents may come from teaching and research institutions in France or abroad, or from public or private research centers.
L'archive ouverte pluridisciplinaire HAL, est destinée au dépôt et à la diffusion de documents scientifiques de niveau recherche, publiés ou non, émanant des établissements d'enseignement et de recherche français ou étrangers, des laboratoires publics ou privés. 


\title{
Scaling-down lithographic dimensions with block-copolymer materials: 10nm- sized features with PS-b-PMMA.
}

X. Chevalier ${ }^{\mathbf{a}, \mathbf{b}, *}$, C. Nicolet ${ }^{\mathbf{a}, \mathbf{c}}$, R. Tiron ${ }^{\mathbf{b}}$, A. Gharbi ${ }^{\mathbf{b}}$, M. Argoud ${ }^{\mathbf{b}}$, J. Pradelles ${ }^{\mathbf{b}}$, M. Delalande ${ }^{\mathbf{d}}$, G. Cunge ${ }^{\mathbf{d}}$, G. Fleury ${ }^{\mathbf{c}}$, G. Hadziioannou', C. Navarro ${ }^{\mathbf{a}}$

a / ARKEMA FRANCE, Route Nationale 117, BP34- 64170 Lacq, France

b / CEA, LETI, MINATEC Campus, 17 rue des Martyrs, 38054 Grenoble cedex 9, France

c / LCPO-UMR 5629 Université Bordeaux I-CNRS, 33405 Talence cedex, France

d / LTM-CNRS, CEA, LETI, 17 Rue des Martyrs, 38054 Grenoble cedex, France

*contact: xavier.chevalier@cea.fr

\begin{abstract}
:
PS- $b$-PMMA block-copolymers systems synthesized on an industrial scale, and satisfying microelectronic's requirements for metallic contents specifications, are studied in terms of integration capabilities for lithographic applications. We demonstrate in particular that this kind of polymer can efficiently achieve periodic features close to 10 $\mathrm{nm}$. These thin-films can be transferred in various substrates through dry-etching techniques. The self-assembly optimization for each polymer is first performed on free-surface, leading to interesting properties, and the changes in self-assembly rules for low molecular weight polymers are investigated and highlighted through different graphoepitaxy approaches. The enhancements in self-assembly capabilities toward low periodic polymers, as well as the broad range of achievable features sizes render PS- $b$-PMMA system very attractive ones for lithographic CMOS applications. We conclude showing that high- $\chi$ polymers materials developed in Arkema's laboratories can be efficiently used to reduce the pattern's size beyond the ones of PS- $b$-PMMA based BCP's capabilities.
\end{abstract}

Keywords: lithography, block-copolymer, self-assembly, PS-b-PMMA, graphoepitaxy. 


\section{1/ Introduction}

Block-copolymers (BCP) self-assembly has been identified as an interesting alternative or complementary approach to current advanced lithographic techniques (EUV, e-beam, optical 193nm patterning...) in order to further decrease accessible dimensions for microelectronic ${ }^{1}$. Indeed, their abilities to spontaneously form various periodic nanometers-sized structures - such as cylinders or lamellas - render them particularly attractive for dedicated lithographic applications as lines-spaces or contact-holes. Moreover, since several years, researches over BCP's properties highlighted both that the self-assembly may be controlled through guiding pre-patterns (the so-called graphoepitaxy or chemi-epitaxy approaches) in order to efficiently increase its long-range order while the ordered mesostructures inherent to the BCP self-assembly may be formed on a short-time scale compatible with conventional lithographic processes ${ }^{2}$.

However, the self-assembly properties are intimately linked to the chemical nature and architecture of the BCP used. For example, it is well-known that the accessible morphologies of a BCP constituted with three chemically distinct blocks ("ABC" type tri-BCP, "A" "B" and "C" representing each block) are far more numerous when compared to the ones of a simple di-block copolymer". Furthermore, it has been demonstrated that the use of a tri-BCP with an "ABA" architecture (same chemical block at the both extremities of the polymeric chain) may lead to an increase of the time required to reduced defects in the self-assembly when compared to the corresponding simpler di-BCP with the same block-chemistries ${ }^{4}$. A lot of attention has been also paid over the usefulness of BCPs presenting an elevated FloryHuggins interaction parameter (" $\chi$ ”) - i.e. a strong degree of chains repulsion between each block ${ }^{5}$. The high value of the $\chi$ parameter allows the synthesis of BCPs with a lower overall molecular weight, leading directly to smaller BCP features with sharp interfaces between the blocks components. The main drawback to an increased value of the $\chi$ parameter is an additional energetic cost for the self-assembly to take place, though the total energy required is lowered through the use of smaller polymeric chains. Therefore, the self-assembly kinetic, depending on the balance of the couple $\chi$ value/polymer-size, could be reduced for such systems.

Among the various BCP systems reported in the literature, poly(styrene)-block-poly(methylmethacrylate) (noted PS- $b$-PMMA) is a well-studied one and presents interesting properties from a technological point of view. First, styrene and methylmethacrylate monomers can be readily co-polymerised in the same reactor through nitroxide mediated polymerisation - i.e. without metal catalyst - in order to obtain a random copolymer (noted PS-r-PMMA) with precise composition. This property is extremely valuable since it is limited to only few monomeric systems, and because randoms copolymers are used as effective "neutral" passivation layer on substrates to orient properly the BCP features ${ }^{6}$. Moreover, the intrinsic chemical nature of both PS and PMMA monomers allows under appropriate conditions to synthetize a BCP with well-controlled architecture and composition, and low dispersity of chains distribution. In addition, at elevated temperatures the surface tension of both PS and PMMA are almost equivalent, providing therefore a natural "neutral" interaction between ambient air and the BCP. Consequently perpendicular BCP features can be obtained without the use of sophisticated systems (solvents atmosphere, vacuum...), which could be potentially prohibitive for lithography. It has also been demonstrated that the self-assembly of a PS- $b$-PMMA system takes place with a simple thermal bake at elevated temperature in a short time, i.e. under similar conditions as classical lithographic processes $^{7}$. Despite those interesting characteristics, a technological debate remains open about the usefulness of this particular system for lithographic applications due to the "small" Flory-Huggins interaction parameter between PS and PMMA, limiting thus the minimum accessible feature's size, though this same "small" $\chi$ favors the self-assembly kinetic of higher molecular-weight polymers.

In this paper we present our studies on the self-assembly of PS- $b$-PMMA BCP systems exhibiting both lamellar and cylindrical morphologies. Those BCPs are synthesized by Arkema, using anionic polymerization reaction at a semiindustrial scale with a well-controlled reproducibility and low dispersity for various molecular weights and compositions. Self-assembly processes are carefully optimized over a wide range of polymers molecular weights, in order to obtain processes largely independent of the thin thickness while satisfying the stringent lithographic requirements (short times processes, metal-free polymers...). We show in particular that low molecular weight PS- $b$ PMMA systems can reach perpendicular features with critical dimensions as low as $\sim 10 \mathrm{~nm}$, rendering therefore such materials very attractive down to the corresponding technological node. Conversely, we show that high molecular weight BCP can be also synthesized efficiently to achieve features with natural periods close to $50 \mathrm{~nm}$, rendering this kind of polymers interesting for an early introduction of BCP technology into current logical-nodes steps. The different morphologies and molecular weights are integrated through various graphoepitaxy approaches to demonstrate the usefulness of those systems for lithography, and the characteristics of each ones are highlighted mainly through the formation of macro-sized polymeric crystals. To further illustrate the usefulness of such polymers for the 
microelectronic, different PS- $b$-PMMA pattern-sizes are transferred in the silica, silicon, or lithography-dedicated substrates through dry-etch techniques. Finally, we show that high- $\chi$ polymers materials developed in our laboratories can be efficiently used to reduce the pattern's size beyond the ones of PS-b-PMMA based BCP's capabilities.

\section{2/ Experimental}

PS- $b$-PMMA and PS-r-PMMA copolymers were synthesized by Arkema, under the tradename Nanostrength EO ${ }^{\circledR}$. The materials were used as received. Microelectronic grade Propylene Glycol Methyl Ether Acetate (PGMEA) used in this study was purchased from Rohm\&Haas ${ }^{\mathrm{TM}}$ and used as received.

Guiding patterns were generated using an e-beam HSQ (hydrogen silsesquioxane) negative tone resist XR-1541 E-Beam Resist, from Dow Corning ${ }^{\circledR} .40 \mathrm{~nm}$ resist thin film was spin-coated on $300 \mathrm{~mm}$ silicon wafers on a RF3 Sokudo track. E-beam lithography was performed using a Vistec VSB 3054DW device. The exposure utilizes an accelerating voltage of $50 \mathrm{kV}$ and an exposure dose of $1350 \mu \mathrm{C} / \mathrm{cm} 2$. After exposure samples were developed for 5 minutes using TMAH at $2.38 \% w t$, and then baked at $215^{\circ} \mathrm{C}$ for $60 \mathrm{~s}$.

PS- $b$-PMMA and PS-r-PMMA powders were dissolved in PGMEA to obtain 1-2\% wt solutions. The PS- $r$ PMMA solution was spin-coated on $3 \times 3 \mathrm{~cm}$ silicon (100) pieces or pre-patterned samples at $700 \mathrm{rpm}$ to obtain thin films of $\sim 90 \mathrm{~nm}$ thickness and then annealed at $230^{\circ} \mathrm{C}$ for $10 \mathrm{~min}$ to ensure the chemical grafting. Afterwards, the substrate was thoroughly washed in PGMEA to remove the ungrafted materials. PS- $b$-PMMA solutions were spin-coated on the top of the PS-r-PMMA brush layer and annealed on a hot-plate at elevated temperature to allow the self-assembly process to take place. Samples with different polymer film thicknesses were generated depending of the processing parameters such as spin-coating speed or the initial solutions concentration. In a last step the PMMA domains were selectively removed by acetic acid development and/or $\mathrm{O}_{2}$ plasma treatment.

The development of the etching processes (random copolymer removal, $\mathrm{SiO}_{2}$ mask opening and silicon etching) was performed in an industrial etcher (DPSTM from Applied Materials). The DPS is an inductively coupled high density plasma source where both the source antennae and the bottom electrode are powered. The source consists of a ceramic dome with a rf three dimensional coil configuration around it. The bottom electrode is powered trough a capacitive coupling at a slightly higher frequency $(13.56 \mathrm{MHz})$ than the source $(12.56 \mathrm{MHz})$. The source power (Ws) controls the ion and reactive neutral species densities, while the bottom power $(\mathrm{Wb})$ controls the energy of ions impinging onto the wafer surface. The lower electrode is a monopolar electrostatic chuck (ECS) and the wafer temperature is kept at $50^{\circ} \mathrm{C}$ by helium backside cooling on the ECS chuck. The $\mathrm{Al}_{2} \mathrm{O}_{3}$ chamber wall temperature is maintained at $80^{\circ} \mathrm{C}$ by a heat exchangers while the ceramic dome is air cooled when the plasma is on, and is heated by lamps when the chamber is idle, providing a constant temperature of $60^{\circ} \mathrm{C}$.

Top-view SEM pictures were acquired on a CD-SEM H9300 from Hitachi.

\section{3/ Results and discussion}

\section{1/ Synthesis and characterizations}

PS- $b$-PMMA with various molecular weights and morphologies (cylindrical or lamellar polymers) used in this study were synthesized in Arkema's facility using sequential anionic polymerization reaction, with a production capacity based on an industrial level. Even with such high-scale type production, the polymer macromolecular characteristics (molecular weight, composition and dispersity) as shown in Figure 1 are very well controlled, and their physico-chemical characteristics match well with those of classical laboratory-made BCP where only few grams of product per batch may be obtained.

The semi-continuous process flow, in combination with the sequential polymerization reaction, used to synthesize the BCP allows for a high-flexibility designed polymeric architecture, in terms of morphologies, composition, and molecular weight. Indeed, this process allows the fine tuning of the final BCP molecular weight and composition during the polymerization reaction, to obtain in fine different BCPs starting from the same living PS precursor if needed (Figure 2). This flexibility in the BCP design contributes for the selection of polymeric systems showing improved physico-chemicals characteristics as regards to the self-assembly properties.

After purification of the raw materials, the level of residual metallic contamination is investigated with Inductively-Coupled-Plasma-Mass-Spectrometry (ICP-MS) analysis. Typical spectrum representing the content of various metallic cations species in the BCP is shown in Figure 3. Striking evidence in this spectrum is that the metallic 
ions content for this kind of BCPs is far below the ones coming from others commercially available polymers. Furthermore, since the content is inferior to $10 \mathrm{ppb}$ for each studied cation for a solution at 2\% wt. of BCP in PGMEA, Arkema's BCPs fully satisfy the current lithographic specifications for CMOS dedicated technologies without any contamination risks for $300 \mathrm{~mm}$ tracks. Future improvements in the purification process will even lead to lower metallic ions contents, in order to satisfy harsher specifications.

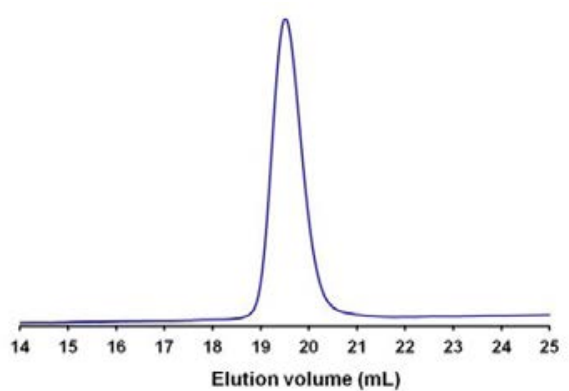

Figure 1: Typical size exclusion chromatography (SEC) curve of a PS$b$-PMMA BCP, synthesized on an industrial scale, exhibiting a molecular chain dispersity of 1.07 .

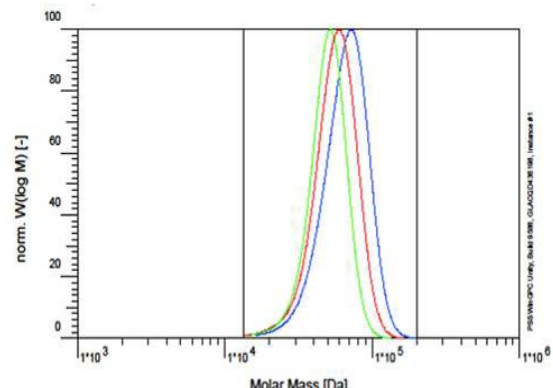

Figure 2: GPC curves of three BCPs with different molecular weights, synthesized from the same PS-living block.

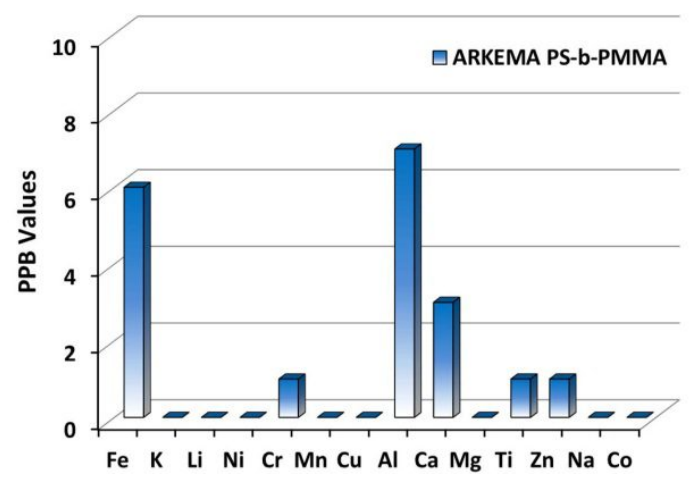

Figure 3: ICP-MS study showing the low metallic contamination level of PS- $b$-PMMA BCPs after purification.

\section{2/ Self-assembly optimization}

PS- $b$-PMMA copolymers were designed to target both cylindrical and lamellar morphologies and to obtain a large range of periods. For both morphologies, the self-assembly process (film thickness, surface-energy, temperature...) was tuned to achieve fully perpendicular features without defects. BCP's periods were determined through Fast Fourier Transform (FFT) or nearest-neighbors-distance measurements starting from calibrated SEM pictures.

For the cylindrical BCPs used in this study the smallest period reached at this time was as low as $22 \mathrm{~nm}$, whereas the largest BCP assembly exhibits a period of $51 \mathrm{~nm}$ with a progressive increment for the BCP period over the range of molecular weight (Figure 4). In the case of lamellar BCPs, the smallest period obtained was $19 \mathrm{~nm}$ (leading to CD inferior to $10 \mathrm{~nm}$ ), and the largest one reached $37 \mathrm{~nm}$ (Figure 5). For both morphologies, the periods shown here are for sure not the ultimate ones that PS- $b$-PMMA based-polymers may achieve, and current works are in progress in order to lower and enlarge further the accessible periods with these polymers. Anyway, both lamellar and cylindrical periods reported here demonstrate that this kind of polymers may be efficiently used as thin films to create nanolithographic masks with CDs close to $10 \mathrm{~nm}$, rendering such systems attractive candidates down to this corresponding technological node. It is interesting to notice also that fully perpendicular cylindrical features can be obtained for periods close to 51 $\mathrm{nm}$. Such high polymeric periods are potentially interesting for an early introduction of BCP self-assembly in lithographic applications (for contact-shrink ones as example) in the current nodes. 


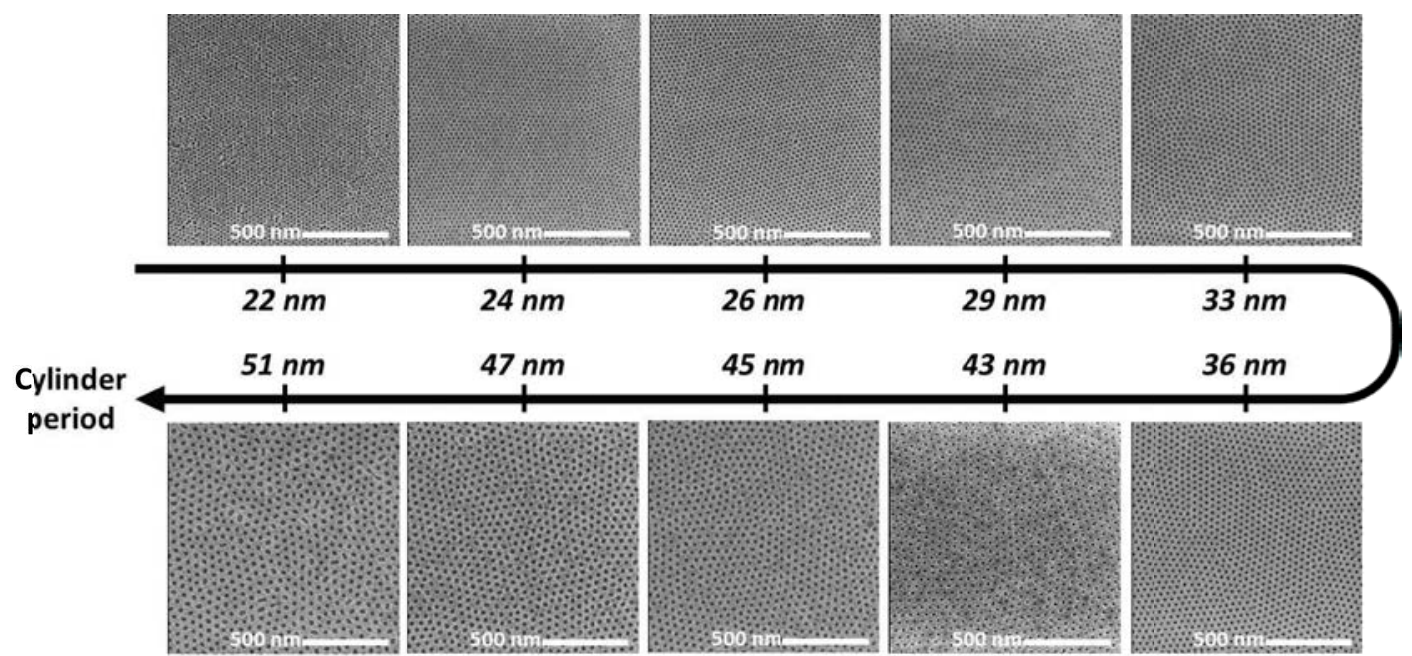

Figure 4: SEM top-view pictures illustrating perpendicular self-assembly of various PS- $b$-PMMA cylindrical BCPs (PMMA cylinders in a PS matrix) with periods ranging from $22 \mathrm{~nm}$ up to $51 \mathrm{~nm}$.

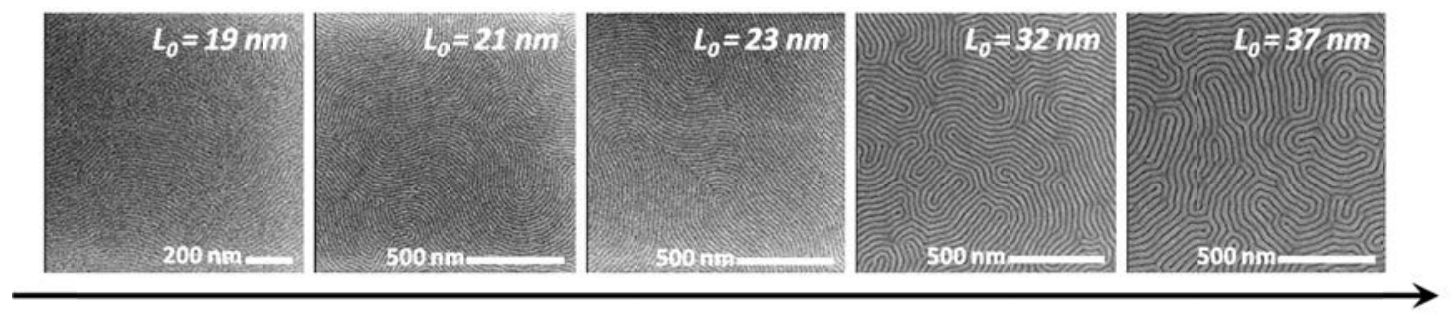

Lamellar block-copolymer period

Figure 5: SEM pictures showing the perpendicular self-assembly of lamellar BCPs with periods ranging from $19 \mathrm{~nm}$ up to $37 \mathrm{~nm}$.

The self-assembly process parameters of all polymers were optimized in order to fit with current lithographic process constraints (short PS-r-PMMA grafting time, short bake-time, temperatures and solvent compatible with $300 \mathrm{~mm}$ track...). The fine tuning in BCP's composition during the synthesis allowed us to select PS-b-PMMA systems with very interesting properties. Indeed, some of them exhibit for example very weak dependence toward the BCP film-thickness: the BCP self-assembly was performed with a $5 \mathrm{~nm}$ increment in film thickness as shown in Figure 6 (all the pictures are not reported here), starting from $20 \mathrm{~nm}$ up to $270 \mathrm{~nm}$, and the polymer exhibits a fully perpendicular cylindrical structure without any defects (in-plane cylinders...) over the whole range of thickness. In the same way, another interesting characteristic for lithographic applications may be the low sensibility of the perpendicular self-assembly toward the surface-energy (PS-r-PMMA composition used for substrate modification). To illustrate this property, the SEM pictures displayed in Figure 7 show a polymer with perpendicular cylindrical structure for very different PS contents in PS-r-PMMA (the variation in PS content between "\%PS1" and "\%PS3" is close to $25 \%$ ), although few defects appear for specific BCP film-thickness. This can be used to alleviate the use of randoms copolymers with a specific composition to obtain perpendicular self-assembled structure, enlarging therefore the BCP process-window available for lithography. 


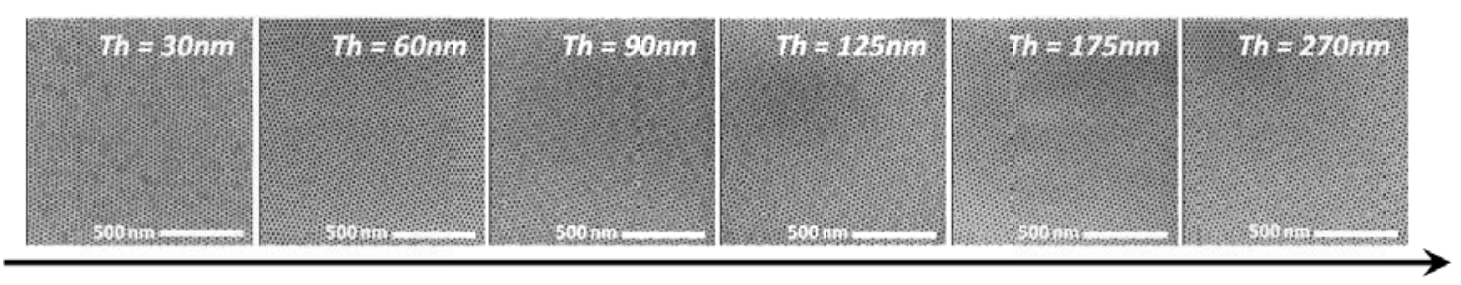

Block-copolymer film thickness

Figure 6: Scheme of SEM pictures illustrating the weak dependence between a BCP and its film thickness.

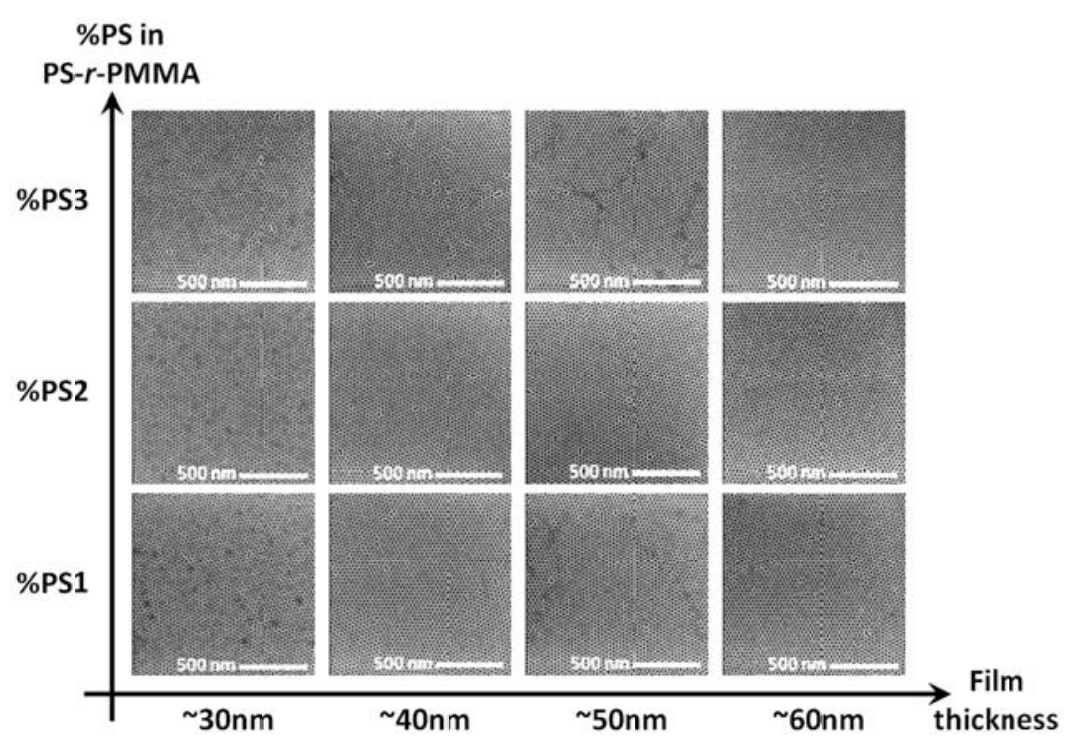

Figure 7: Self-assembly of a BCP with different film-thickness on various PS-ir-PMMA with different PS contents showing that weak surface-energy dependence may be achieved.

The BCP self-assembly process optimization becomes extremely important for low molecular-weight polymers. Indeed, the order-disorder transition temperature $\left(\mathrm{T}_{\mathrm{ODT}}\right)$ for these particular systems are quiet low and the hightemperature bake inherent to the self-assembly process cannot be maintained for small PS- $b$-PMMA without presenting the risk to exceed the $\mathrm{T}_{\mathrm{ODT}}$ of the system, leading to featureless structures. It is rather an advantage for PS- $b$-PMMA polymers self-assembly toward small lithographic dimensions since the decrease in bake temperatures will contribute to the reduction in process costs. This is not the sole advantage in the use of small PS-b-PMMA polymers for lithography, some other ones, linked to graphoepitaxy approach, will be described in the section 3.3. The dependence of the self assembly process as regards to the temperature is illustrated in the Figure 8 , where the classical $240-250^{\circ} \mathrm{C}$ (or more) process temperature has to be modified for this $19 \mathrm{~nm}$ period lamellar polymer: at constant process-time, both $160^{\circ} \mathrm{C}$ and $200^{\circ} \mathrm{C}$ bakes show poorly ordered perpendicular structures, while well-ordered lamellas appear for a temperature close to $180^{\circ} \mathrm{C}$. The fact that the lamellas are not well featured at $160^{\circ} \mathrm{C}$ is due to a slow self-assembly kinetic (due to the high $\mathrm{Tg}$ of PMMA and PS), while at $200^{\circ} \mathrm{C}$ (and $190^{\circ} \mathrm{C}$ ) the PS and PMMA chain segments become miscible leading to the loss of the order parameter. We therefore situated the $\mathrm{T}_{\mathrm{ODT}}$ of this polymer between 180 and $190^{\circ} \mathrm{C}$ and recently demonstrated this value by DMA measurements at low shearing rate.

It is interesting to note also that $\mathrm{T}_{\mathrm{ODT}}$ of PS- $b$-PMMA BCPs are now accessible with these small polymers, whereas it is usually located above the degradation point for more classical BCPs. The location of the $\mathrm{T}_{\mathrm{ODT}}$ for wellcalibrated molecular weights and morphologies will lead to interesting information sets for self-assembly of PS- $b$ PMMA-based polymers, but this is beyond the scope of this study and these results will be reported elsewhere. 


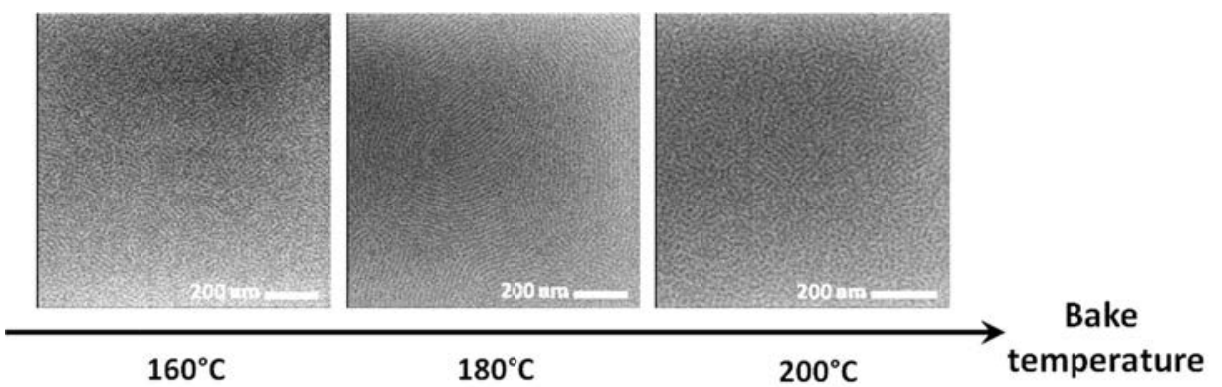

Figure 8: Self-assembly of a low molecular weight lamellar PS- $b$-PMMA BCP presenting a $\mathrm{T}_{\mathrm{ODT}}$ close to $190^{\circ} \mathrm{C}$, illustrating the fact that the process parameters must be carefully tuned to reach an optimal order.

A two-steps dry-etching procedure was already reported in previous publications in order to transfer the BCP mask in the bulk silicon or substrates of interest for lithography such Spin-on-Carbon (SOC), through a sacrificial silica hard-mask methodology leading to nice aspect-ratio features ${ }^{8,9}$. This procedure contains three main steps: a first step consists in the PS-r-PMMA layer removal with a short $\mathrm{Ar} / \mathrm{O}_{2}$ plasma, followed by the transfer of the BCP features in the silica hard-mask layer with a fluorocarbon based-plasma, and these silica structures are finally transferred in the bulk silicon with a $\mathrm{HBr} / \mathrm{Cl}_{2} / \mathrm{O}_{2}$ plasma. The same approach is kept for the SOC substrate: after the step of brushes opening with a $\mathrm{Ar} / \mathrm{O}_{2}$ plasma, the features are transferred in the SiARC hard-mask with fluorocarbon chemistry, and then in the SOC with a $\mathrm{O}_{2}$ plasma. The Figure 9(a) and Figure 9(b) show that $36 \mathrm{~nm}$-period BCP can be efficiently transferred in bulk silicon as well as in SOC substrate respectively with high aspect-ratio features. The Figure 9(c) shows that the 28 nm-period BCP mask may be successfully transferred in the $10 \mathrm{~nm}$-thick silica hard-mask, leading to features with a mean CD close to $11 \mathrm{~nm}$. Although the dry transfer process is still under improvement, one has to mention that the PS-rPMMA opening step is critical to avoid an exceeding enlargement of the final $\mathrm{CD}^{10}$. Anyway, as the BCP's period used here is not the smallest one available, final CD (after etch-transfer) could reach efficiently dimensions close to or below $10 \mathrm{~nm}$ with PS-b-PMMA BCPs.

(a)

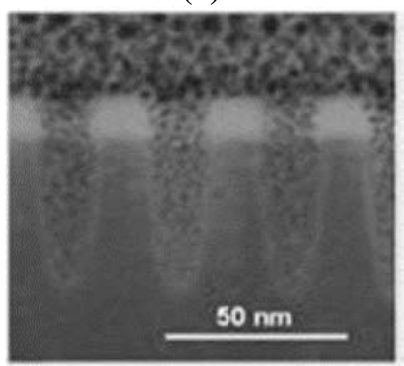

(b)

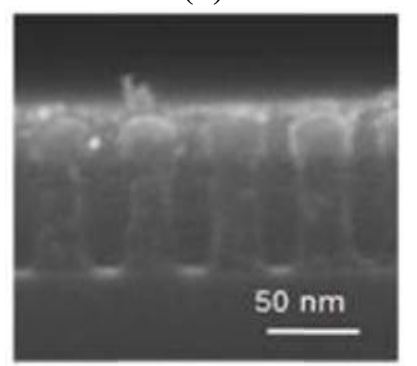

(c)

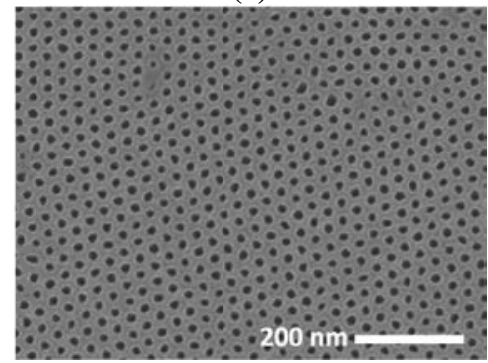

Figure 9: Left and middle pictures: $36 \mathrm{~nm}$-period BCP transferred in bulk silicon and SOC substrate respectively (the upper white areas are the remaining $\mathrm{SiO}_{2}$ and $\mathrm{SiARC}$ hard-masks respectively). Right picture: $10 \mathrm{~nm}$-thick silica hard-mask after dry etch-transfer of a PS-b-PMMA BCP leading to features with CD close to $11 \mathrm{~nm}$.

\section{3/ Graphoepitaxy approaches}

Various approaches have been studied since several years in order to improve the correlation length of the BCP self-assembly. Among them, the so-called chemi-epitaxy, using patterns based on chemical contrasts ${ }^{11}$, and the graphoepitaxy $^{12}$, which uses physical templates (e.g. lithographic resists hard-walls), are both well-known methods to guide efficiently the BCP self-assembly. The improvement of these two techniques leads to their recent introduction in $300 \mathrm{~mm}$ tracks as BCP tests structures, chemi-epitaxy for line-space applications ${ }^{13}$, graphoepitaxy for contact-hole shrink applications in lithography ${ }^{14}$. Both of these methods may present some issues: chemi-epitaxy offers for example some ease of processing, but suffers somewhat of a low capacity in density multiplication of pre-patterns since there is no lateral constrain to guide the BCP, while graphoepitaxy presents this efficient lateral strain, but the pre-patterns have to resist to solvents or high-temperature bake used for the BCP process. 
Our previous studies focused on the graphoepitaxy approach since it starts from an already existent pre-pattern generated with well-established conventional lithographic techniques (optical or mask-less), and that the BCP selfassembly process is compatible with many substrates/materials ${ }^{15}$. We also demonstrated that this method can be used efficiently to create real two-dimensional macroscopic BCP crystals with long-range order (like those obtained via chemi-epitaxy) if commensurability conditions between the BCP-lattice and the pre-pattern are well respected ${ }^{16}$.

For practical reasons the pre-patterns used in this study were made with e-beam lithography with a HSQ-based resist, though self-assembly rules depicted here would work for others writing methods and materials. The SEM pictures in the Figure 10 show "classical" (i.e. BCP film-thickness below the height of the HSQ lines) graphoepitaxy samples obtained with two lamellar BCPs having different intrinsic period. An important point to notice is that the self-assembly processes used for these two samples are entirely equivalent; the bake time used was deliberately short (few minutes) in order to fit those of usual lithographic materials. A striking difference between these two samples is the quality of the BCP self-assembly. Indeed, the Figure 10(a) shows the assembly of the high molecular weight BCP where many defects are visualized (jogs, broken BCP lines...) although the commensurability conditions were respected in order to obtain three standing-up lamellas. Conversely, the picture resulting from the self-assembly of the small period BCP (Figure 10(b)) shows eight perfectly aligned standing-up lamellas without defects. Such contrast between the assemblies obtained with the two polymers is solely due to the difference in polymer molecular weight: the smaller the polymer is, the faster the self-assembly kinetic is (the faster it reaches its equilibrium state). Although not reported here, the same effect has been observed in the case of standing-up cylindrical BCP features. Therefore, the use of small molecular weight PS-b-PMMA polymers could be extremely valuable and interesting for lithography since it allows for faster selfassembly processes (phase separation, kinetic of defects reduction...) leading to an increased density multiplication potential.

(a)

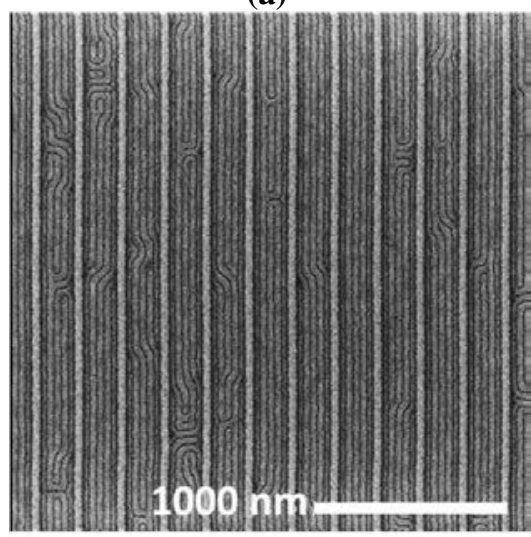

(b)

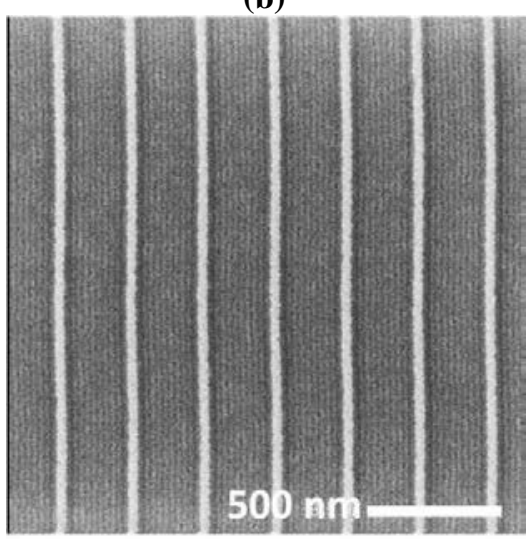

Figure 10: Graphoepitaxy of two lamellar BCPs having different molecular weights, illustrating the increase in ordering for a low molecular weight BCP (b) when the same self-assembly process with a higher molecular weight (a) one is done (white lines: HSQ template).

The "classical" graphoepitaxy approach leads to a one dimensional long-range order, each template being independent from the others, and each resist line being separated from the next one by few hundred nanometers. The 1D order may be transformed into a 2D order by over-layering the polymer film thickness above the pattern height and controlling carefully the commensurability conditions between the pattern's size and the BCP. This method has been demonstrated with perpendicular cylindrical BCP features, leading to perfectly ordered macroscopic polymer crystals ${ }^{16}$.

We show here that this particular method can be used also efficiently with small molecular weights cylindrical BCPs having CD close to $10 \mathrm{~nm}$. The benefit in the use of small molecular weight BCP is an increase in the degree of freedom concerning the pre-pattern design. Indeed, as in the case of "classical" graphoepitaxy, BCPs having small period tolerate wider patterns and wider grooves without losing a perfect order. Since here the defect level occurs primarily on the top of the patterns (no lateral strain for the BCP: the order is imposed by the pattern width and the polymer in the grooves), the assembly of a low molecular weight BCP allows for higher pattern's width to be used without loss of ordering. This property is illustrated on the Figure 11(b), where 6 rows of cylinders are present on the top of the HSQlines with a high degree of order for the macro-crystal obtained. 
Another interesting case is the combination of this method with lamellar BCPs. Indeed, the SEM pictures displayed in Figure 11 (c) and Figure 11(d) show that an increase in the film thickness of lamellar BCP above the prepattern height leads to a 2D long-range order: both lamellas in the grooves and the resist lines guides the lamellas located above the HSQ lines, providing in this way a macroscopic sized BCP crystal over the whole pre-patterned area. As seen for cylindrical BCPs, the use of a low molecular weight lamellar BCP leads to more freedom in the pre-pattern design due to the enhancement in their self-assembly kinetic.

(a)

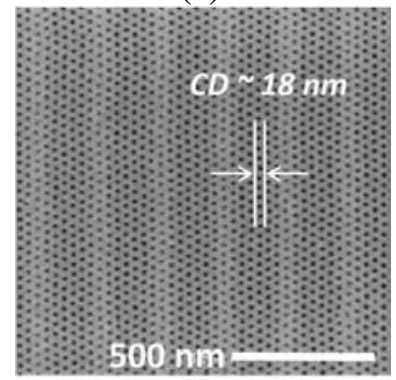

(b)

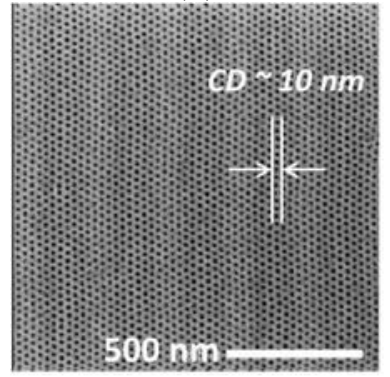

(c)

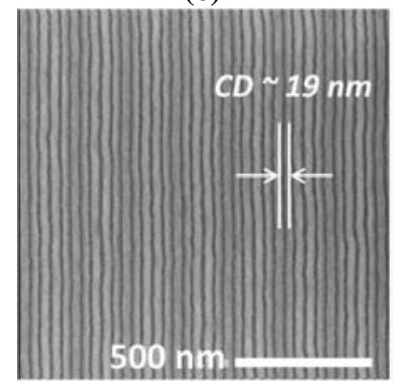

(d)

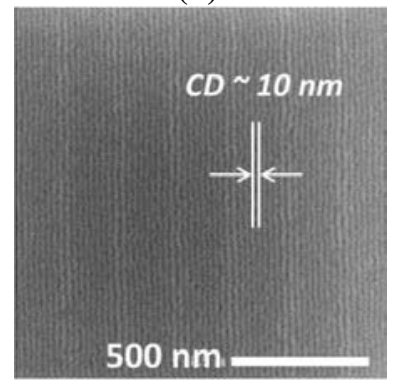

Figure 11: Macroscopic-sized BCPs crystals obtained from a high molecular weight cylindrical BCP (a) and a low molecular weight cylindrical BCP (b), a high molecular weight lamellar BCP (c) and a low molecular weight lamellar BCP (d) (lighter grey areas: BCPs above the HSQ-resist lines).

One promising application for BCP to find a place in lithographic industrial processes is the contact-shrink approach ${ }^{17}$ : starting from a large round pre-pattern, the BCP fills this hole with one or several perpendicular cylinders in the middle. Given that current advanced lithographic tools enable the generation of pre-pattern CD around 60nm, and that the CD of a BCP cylinder fitting this pattern is around 20nm, it would allows in fine to divide the initial pattern's size at least by three, leading therefore to an efficient decrease in the initial hole size for vias application. We show here that PS- $b$-PMMA polymers properties are well adequate for this kind of application, since the pre-pattern's size may range from $\sim 70 \mathrm{~nm}$ down to $45 \mathrm{~nm}$ with different polymers, without pushing the BCP system toward its lowest limits (Figure 12). One should refer the reader in another of these proceeding for further details about the potential of such BCPs for this particular application ${ }^{14}$.
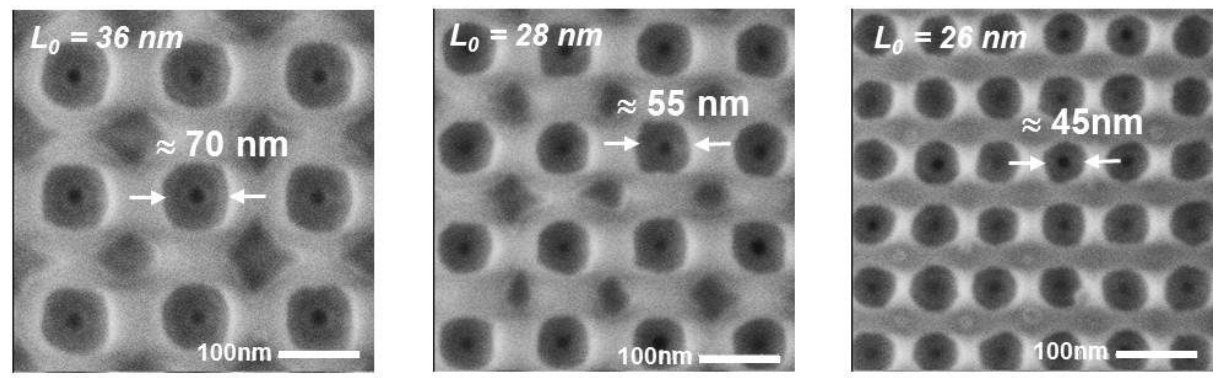

Figure 12: Various pre-patterned contact-hole sizes shrunk with different PS- $b$-PMMA BCPs having periods of 36, 28 and $26 \mathrm{~nm}$ respectively.

\section{4/ High- $\chi$ materials}

Despite PS- $b$-PMMA polymers present interesting properties, achievable CDs with this system will not decrease far below $10 \mathrm{~nm}$ due to its relatively low value of Flory-Huggins parameter. To address this specific problem, BCP chemical systems with higher $\chi$ values have to be developed, along with their self-assembly processes. The SEM pictures displayed in the Figure 13 demonstrate that BCP features close to $7 \mathrm{~nm}$ may be reached with such new polymer systems, and that they can be efficiently guided with traditional graphoepitaxy approach. These results illustrate well the usefulness and capabilities of BCP materials to scale down dimensions of future interest for lithographic applications. 

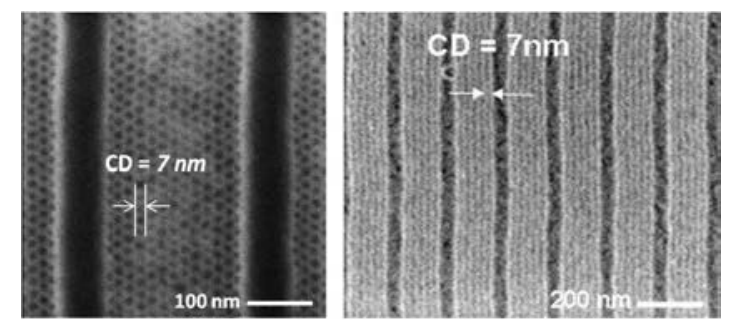

Figure 13: SEM pictures of high- $\chi$ BCPs self-assembled within graphoepitaxy approach (HSQ templates), leading to features down to $7 \mathrm{~nm}$.

\section{4/ Conclusions}

We have shown in this paper that PS-b-PMMA copolymers systems can be produced efficiently at the industrial scale in a controlled manner with well defined architectures, without metallic contaminant, to fulfill the future needs of microelectronic. The interest of such BCP system relies on the broad range of achievable periodic features exhibiting increased self-assembly capabilities when moving toward low molecular weight polymers. Despite its "small" $\chi$ value, this system is able to produce CDs close to $10 \mathrm{~nm}$ and the transfer in the substrate of these features can be done with conventional dry-etching techniques, although the process has still to be improved. Further CDs scaling down, below 10 $\mathrm{nm}$, can be achieved efficiently with high- $\chi$ BCP materials from Arkema.

\section{Acknowledgements}

The authors thank J. Bustos and J. Belledent for the design of HSQ-based templates. This work has been performed under the framework of the IDEAL program involving the different partners.

\section{References}

[1] http://www.itrs.net/Links/2011ITRS/2011Chapters/2011ERM.pdf

[2] H.-C. Kim \& al., Chem. Rev. 2010, 110, 146.

[3] W. Zheng \& al., Macromolecules, 1995, 28, 7215.

[4] S. Ji \& al., ACS Nano, 2012, 6 (6), 5440.

[5] Y. S. Jung \& al., Nano Lett., 2007, 7 (7), 2046.

[6] P. Mansky \& al., Science 1997, 275, 1458.

[7] A. M. Welander \& al., Macromolecules, 2008, 41 (8), 2759.

[8] X. Chevalier \& al., Proc of SPIE 2011, 7970.

[9] T. Chevolleau \& al., Proc of SPIE 2012, 8328-20.

[10] X. Gu \& al., Adv. Mater., 2012, 24, 5505.

[11] S. O. Kim \& al., Nature, 2003, 424, 411.

[12] R.A. Segalman \& al., Adv. Mater., 2001, 13, 1152.

[13] C. Bencher \& al., Proc. of SPIE 2011, 79700F-4.

[14] R. Tiron \& al., Proc of SPIE 2013, 8680-37.

[15] R. Tiron \& al., Proc of SPIE 2012, 8323.

[16] R. Tiron \& al., JVST B, 2011, 29, 06F206.

[17] J. Y. Cheng \& al., ACS Nano, 2010, 4 (8), 4815. 